\title{
M-current preservation contributes to anticonvulsant effects of valproic acid
}

\author{
Hee Yeon Kay, ${ }^{1}$ Derek L. Greene, ${ }^{1}$ Seungwoo Kang, ${ }^{1}$ Anastasia Kosenko, ${ }^{1}$ and Naoto Hoshi ${ }^{1,2}$ \\ 'Department of Pharmacology and 2Department of Physiology and Biophysics, University of California, Irvine, Irvine, California, USA
}

\begin{abstract}
Valproic acid (VPA) has been widely used for decades to treat epilepsy; however, its mechanism of action remains poorly understood. Here, we report that the anticonvulsant effects of nonacute VPA treatment involve preservation of the M-current, a low-threshold noninactivating potassium current, during seizures. In a wide variety of neurons, activation of Gq-coupled receptors, such as the $\mathrm{m} 1$ muscarinic acetylcholine receptor, suppresses the $\mathrm{M}$-current and induces hyperexcitability. We demonstrated that VPA treatment disrupts muscarinic suppression of the M-current and prevents resultant agonist-induced neuronal hyperexcitability. We also determined that VPA treatment interferes with M-channel signaling by inhibiting palmitoylation of a signaling scaffold protein, AKAP79/150, in cultured neurons. In a kainate-induced murine seizure model, administration of a dose of an M-channel inhibitor that did not affect kainate-induced seizure transiently eliminated the anticonvulsant effects of VPA. Retigabine, an M-channel opener that does not open receptor-suppressed M-channels, provided anticonvulsant effects only when administered prior to seizure induction in control animals. In contrast, treatment of VPA-treated mice with retigabine induced anticonvulsant effects even when administered after seizure induction. Together, these results suggest that receptor-induced $\mathrm{M}$-current suppression plays a role in the pathophysiology of seizures and that preservation of the $\mathrm{M}$-current during seizures has potential as an effective therapeutic strategy.
\end{abstract}

\section{Introduction}

Valproic acid (VPA) is used to treat epilepsy and bipolar disorder (1). VPA is known to have multiple pharmacological actions, such as prolongation of sodium-channel inactivation (2), elevation of GABA content in the brain (3), and inhibition of HDAC activity (4). However, underlying mechanisms for the anticonvulsant effect of VPA are still unclear.

The M-current is a low-threshold noninactivating voltagegated potassium current generated by tetrameric channels composed of various combinations of subunits encoded by the KCNQ gene family $(K C N Q 2,3,4$, and 5) $(5,6)$. A recent study showed that the Kv7.2/KCNQ2 is the dominant subunit in pyramidal neurons in the hippocampus (7). Various neurotransmitters that activate Gq-coupled receptors, including acetylcholine, suppress the $\mathrm{M}$-current and induce neuronal hyperexcitability. Accordingly, loss-of-function mutations in KCNQgenes are known to cause epilepsy and encephalopathy $(5,8)$, and M-channel openers are used to treat epilepsy $(9,10)$.

Intensive studies have been conducted to elucidate the molecular pathway mediating M-current suppression induced by neurotransmitters, especially by the muscarinic action of acetylcholine $(5,6,11,12)$. These studies revealed that multiple signaling pathways converge at the $\mathrm{M}$-channel complex (12-15). One mechanism is through the depletion of phosphatidylinositol 4,5-bisphosphate (PIP2), an essential cofactor for ion conduction. In this mechanism, activation of Gq-coupled receptors activates phospholipase C

Authorship note: Hee Yeon Kay and Derek L. Greene contributed equally to this work. Conflict of interest: The authors have declared that no conflict of interest exists. Submitted: October 28, 2014; Accepted: July 30, 2015.

Reference information: J Clin Invest. 2015;125(10):3904-3914. doi:10.1172/JCI79727.
(PLC), which consumes PIP2 leading to its loss from KCNQ subunits. PIP2 deficient M-channel cannot conduct potassium ions, since PIP2 is required to prevent collapse of the ion-conducting pore (16). Another mechanism is mediated by PKC phosphorylation of the KCNQ2 subunit, which reduces PIP2 efficacy. Upon activation of $\mathrm{m} 1$ muscarinic receptor, PKC bound to the KCNQ2 subunit via A-kinase anchoring protein (AKAP79/150) phosphorylates the KCNQ2 subunit. Phosphorylation of KCNQ2 dissociates calmodulin (CaM), thereby destabilizing KCNQ2-PIP2 interaction and leading to the shutdown of M-channel activity (15).

AKAP79/150 (human AKAP79/rodent AKAP150) is a critical signaling-scaffold protein for the M-channel, which tethers several signaling enzymes, facilitating local signaling events. AKAP79/150 is known to be important not only for the M-channel, but also for glutamate receptors and other ion channels $(14,17,18)$. AKAP150 (Akap5) gene knockout mice show impaired learning and resistance to pilocarpine-induced seizures (19). In addition, recent studies have shown that palmitoylation of AKAP79/150 plays essential roles in cellular signaling $(20,21)$. Palmitoylation is a reversible posttranslational modification, which adds predominantly palmitic acid to selective cysteine residues (22). Palmitoylation is required for neural plasticity, as it targets relevant proteins to lipid rafts, which often creates signaling hot spots (23). Furthermore, changes in neuronal activities induce depalmitoylation (21, 24). Therefore, palmitoylation is gaining greater attention due to its dynamic regulation during signaling events.

We demonstrate that VPA treatment disrupted muscarinic acetylcholine receptor-induced M-current suppression, preventing receptor-induced hyperexcitability in neurons. Consistently, pharmacological blockade of M-current diminished the anticonvulsant effect of VPA but not that of diazepam. We show that reti- 
A SCG neuron VPA-treated SCG neuron

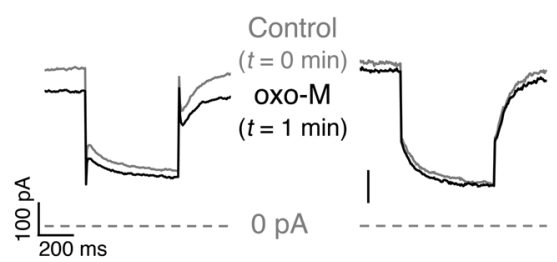

(11)

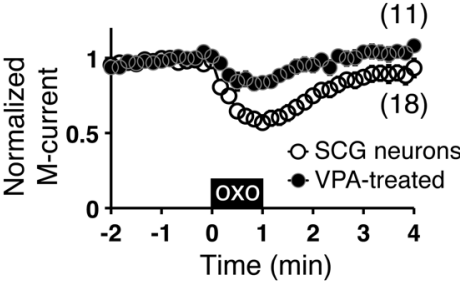

B

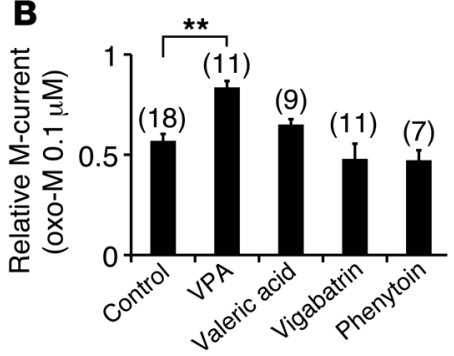

C<smiles>O=C(O)CCCCCO</smiles>

Control

Oxo-M

XE991

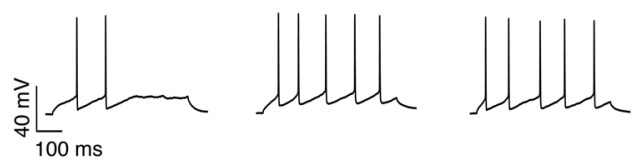

$$
\begin{array}{r}
\begin{array}{r}
\text { VPA-treated } \\
\text { hippocampal } \\
\text { neuron }
\end{array} \\
\mathrm{OH}
\end{array}
$$<smiles>CCCCC</smiles><smiles>CCCC</smiles><smiles>C#CCCCC</smiles>

Valeric acid-treated hippocampal<smiles>CCCCC(=O)O</smiles><smiles>CCCCCC</smiles><smiles>C=CC(C)CC</smiles>

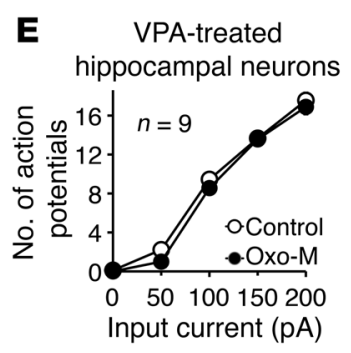

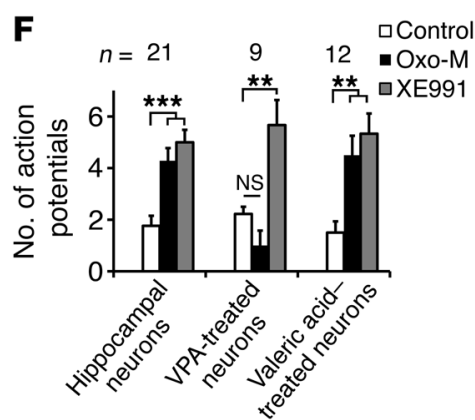

Figure 1. VPA attenuated muscarinic suppression of the M-current and prevented oxo-M-induced hyperexcitability in cultured SCG and hippocampal neurons. (A) Representative voltage-clamp current traces from SCG neurons (top) and pooled results (bottom) showing M-current suppression by $0.1 \mu \mathrm{M}$ oxo-M and its disruption by $500 \mu \mathrm{M}$ VPA pretreatment. Amplitudes of the M-currents are normalized to those at $t=0$. Black box indicates presence of $0.1 \mu \mathrm{M}$ oxo-M. (B) Summary of muscarinic suppression of the M-current at $t=1$ min including results shown in $\mathbf{A}$. Muscarinic suppression is attenuated by $500 \mu \mathrm{M}$ VPA but not by $500 \mu \mathrm{M}$ valeric acid, $100 \mu \mathrm{M}$ vigabatrin, or $100 \mu \mathrm{M}$ phenytoin. ${ }^{* *} P<0.01$ using Kruskal-Wallis one-way ANOVA followed by Dunn's multiple comparisons test. Numbers above bars represent $n$ values. (C) Current clamp traces showing action potential firing in response to 50 pA current injection. Traces from VPA-nontreated (top), $500 \mu \mathrm{M}$ VPA-treated (middle), and $500 \mu \mathrm{M}$ valeric acid-treated (bottom) hippocampal neurons are shown. Control, presence of $1 \mu \mathrm{M}$ oxo-M, or presence of $20 \mu \mathrm{M}$ XE991. (D and E) Input-excitation relationships from hippocampal neurons before (open circle) and after application of oxo-M (filled circle) from nontreated neurons (D) or $500 \mu \mathrm{M}$ VPA-treated neurons (E). (F) Summary of neural firing by 50 pA current injection shown in C-E. ${ }^{* *} P<0.01$ and ${ }^{* *} P<0.001$ using repeated measures one-way ANOVA followed by Tukey's multiple comparisons test. Error bars show \pm SEM.

gabine, an M-channel opener, had anticonvulsant effect only when administered before induction of seizure in control mice. However, in VPA-treated mice, anticonvulsant effect of retigabine was maintained even when administered after seizure induction. We determined that disruption of receptor-induced M-current suppression was caused by reduced palmitoylation of AKAP79/150 by VPA treatment. These findings suggest that M-current suppression plays a role in the pathology of seizures and that disruption of M-current suppression is an integral part of the anticonvulsant mechanism of nonacute VPA treatment.

\section{Results}

VPA prevents muscarinic agonist-induced neuronal hyperexcitability by disrupting M-current suppression. VPA has been used to treat epilepsy and bipolar disorder for several decades (25). Accumulating genomic analyses indicate that these 2 neurological disorders are also linked with KCNQ gene mutations $(5,26)$. The similarity between the 2 motivated us to investigate whether VPA affects M-channel regulation.
Rat superior cervical ganglion (SCG) neurons were used in the initial assessment of VPA effects on the M-current, since M-current regulation has been extensively characterized in this neuronal type and neurons in the CNS are known to use the same regulatory mechanism $(6,27)$. SCG neurons were pretreated with $500 \mu \mathrm{M}$ VPA for 3 days, and $\mathrm{M}$-current response to a muscarinic agonist, $0.1 \mu \mathrm{M}$ oxotremorine-M (oxo-M), was measured. VPA-treated neurons showed reduced muscarinic suppression of the M-current (Figure 1A). In contrast, pretreatment with a structurally similar short-chain fatty acid, valeric acid $(500 \mu \mathrm{M})$, did not affect muscarinic suppression of the M-current (Figure 1B). In addition, other antiepileptic compounds, $100 \mu \mathrm{M}$ vigabatrin or $100 \mu \mathrm{M}$ phenytoin, did not change muscarinic suppression of the M-current (Figure 1B).

We next tested whether VPA also affects M-current-mediated neuronal excitability in central neurons using hippocampal neurons. Cultured rat hippocampal neurons were pretreated with 500 $\mu \mathrm{M}$ VPA or $500 \mu \mathrm{M}$ valeric acid for 3 days. Current clamp recording was used to measure neuronal excitability of these neurons. In control nontreated neurons, 50 pA current injections for 500 
A
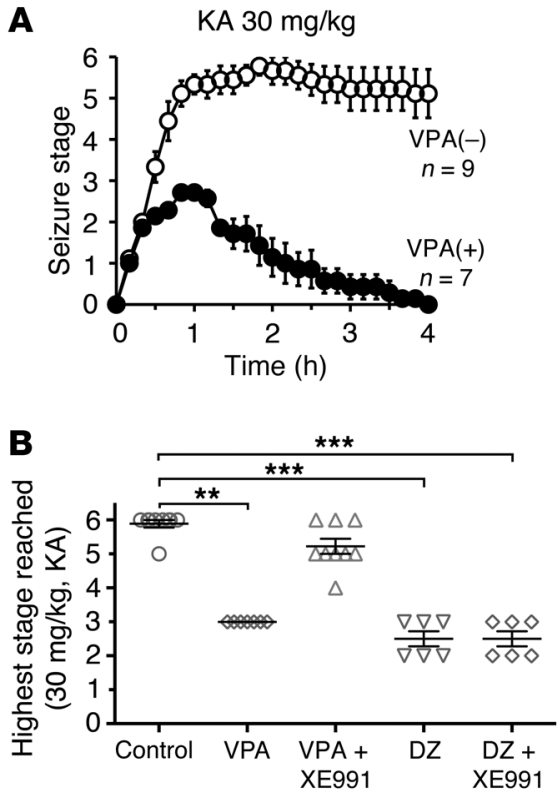
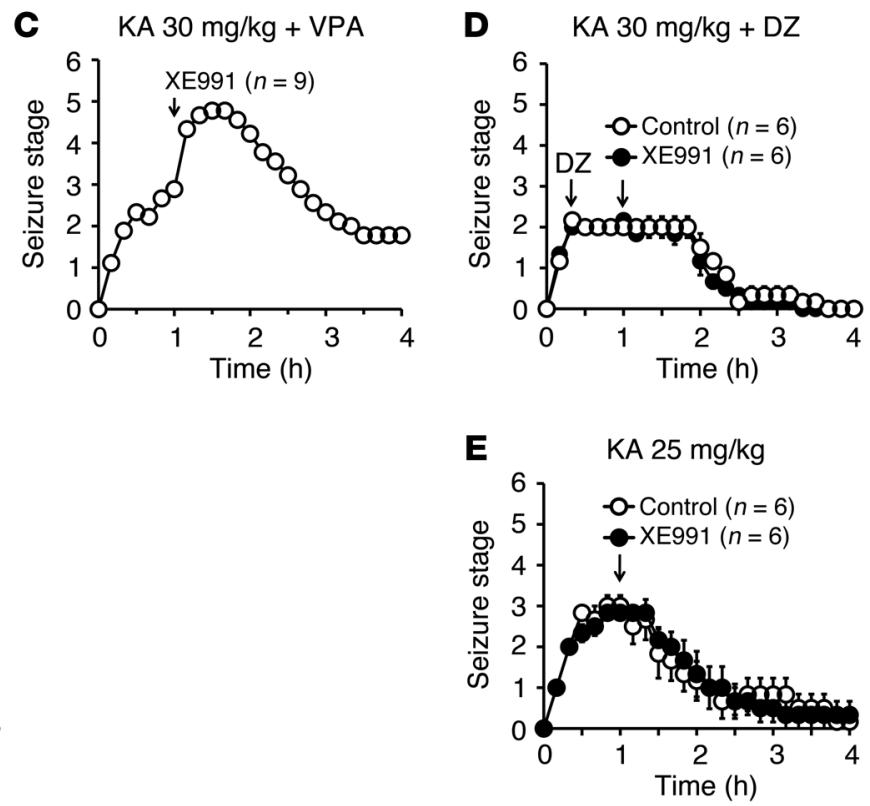

Figure 2. XE991 transiently removed anticonvulsant effect of VPA in kainate-induced seizure in mice. (A) Pretreatment with VPA decreased seizure stages of kainate-induced seizure (KA, $30 \mathrm{mg} / \mathrm{kg}, t=0)$ in adult mice. (B) Distribution of highest seizure stages reached in experiments shown in $\mathbf{A}, \mathbf{C}$, and D. ${ }^{* *} P<0.01$ and ${ }^{* * *} P<0.001$ using Kruskal-Wallis one-way ANOVA followed by Dunn's multiple comparisons test. (C) Administration of XE991 (2 mg/ $\mathrm{kg}$ ) transiently removed anticonvulsant effects of VPA. (D) Anticonvulsant effect of diazepam (DZ, $5 \mathrm{mg} / \mathrm{kg}$ ) was not affected by XE991 (2 mg/kg). (E) XE991 $(2 \mathrm{mg} / \mathrm{kg}$ ) did not affect stage-3 seizure induced by $25 \mathrm{mg} / \mathrm{kg}$ kainate in VPA-nontreated mice. Arrows show time points for injection of indicated compounds. $\mathrm{KA}$ is injected at $t=0$. Error bars show $\pm \mathrm{SEM}$.

ms evoked action potential firing (Figure 1C, top row). Application of $1 \mu \mathrm{M}$ oxo-M increased neuronal firing in control hippocampal neurons (Figure 1C). This oxo-M-induced hyperexcitability was apparent below $150 \mathrm{pA}$ current injections in our conditions (Figure 1D). Similar hyperexcitability could be reproduced by the application of an M-channel inhibitor, XE991 (Figure 1, C and F).

In VPA-treated neurons, we observed normal input-excitation relationships in the quiescent condition (Figure 1, C and E). However, oxo-M application did not increase neuronal excitability, as would be expected from attenuated muscarinic M-current suppression (Figure 1, C and E). The application of XE991 to VPA-treated neurons induced hyperexcitation similar to that in VPA-untreated neurons (Figure 1, C and F). On the other hand, valeric acid-treated neurons did not show any difference from nontreated hippocampal neurons in basal excitability, oxo-Minduced hyperexcitability, or XE991-induced hyperexcitability (Figure 1F). These results suggest that VPA treatment disrupted muscarinic suppression of the M-current and prevented receptor-induced hyperexcitability.

Preserved M-current contributes to anticonvulsant effects of VPA. To test whether disruption of $\mathrm{M}$-current suppression contributes to anticonvulsant effects of VPA, we evaluated the anticonvulsant effect of VPA in kainate-induced seizure model. In WT C57BL/6 mice, $30 \mathrm{mg} / \mathrm{kg}$ kainate induced seizures, which usually lead to generalized convulsions (stage 6, Figure 2, A and B) and often to mortality $(7 / 9$ mice $)$. VPA treatment $(250 \mathrm{mg} / \mathrm{kg} \times 2 /$ day $\times 3.5$ days) diminished the severity to stage 3 (Figure 2, A and B) and prevented mortality $(0 / 7$ mice, $P=0.0032$. Fisher's exact test).

Our in vitro experiments suggest that VPA counteracts receptor-induced M-current suppression. Therefore, we reasoned that
XE991, a voltage-dependent inhibitor of the M-channel (28), should diminish anticonvulsant effects of VPA. XE991 is a unique channel blocker that does not inhibit M-channel when the membrane potential is below $-65 \mathrm{mV}$ (28), which is close to the resting membrane potential of neurons. Hence, it should have minor effects on neurons showing sparse firing. Indeed, administration of XE991 $(2 \mathrm{mg} / \mathrm{kg})$ did not affect basic motor behaviors in mice (Supplemental Figure 1; supplemental material available online with this article; doi:10.1172/JCI79727DS1). However, this dose of XE991 transiently exacerbated kainate-induced seizure in VPAtreated mice reaching stage- 5 seizures (Figure 2C). In contrast, $2 \mathrm{mg} / \mathrm{kg}$ XE991 did not affect either the anticonvulsant effect of diazepam (Figure 2D) or stage-3 seizures in VPA-nontreated mice induced by reduced kainate $(25 \mathrm{mg} / \mathrm{kg}$, Figure $2 \mathrm{E})$. These results suggest that VPA shows anticonvulsant effect through preservation of the M-current. Inversely, since control kainate-induced seizures and diazepam-treated seizures were not affected by XE991, these results also suggest that $\mathrm{M}$-current is suppressed during seizures.

One caveat of the above experiments is a possibility that observed effects of XE991 were derived from off-target inhibition of other potassium channels, such as Kv4 channels (29). To further test whether M-current is involved in seizure pathology and VPA action, we performed a second set of experiments using retigabine as a probe, which does not affect $\mathrm{Kv} 4$ channels (30). Retigabine is a recently approved antiepileptic drug that opens the M-channel by shifting activation voltage close to the resting membrane potential (30). In addition, retigabine has been demonstrated to be ineffective at opening $\mathrm{M}$-channels under receptor-induced suppression (30). We first tested whether VPA treatment modulates the pharmacological action of retigabine in vitro using tran- 
A
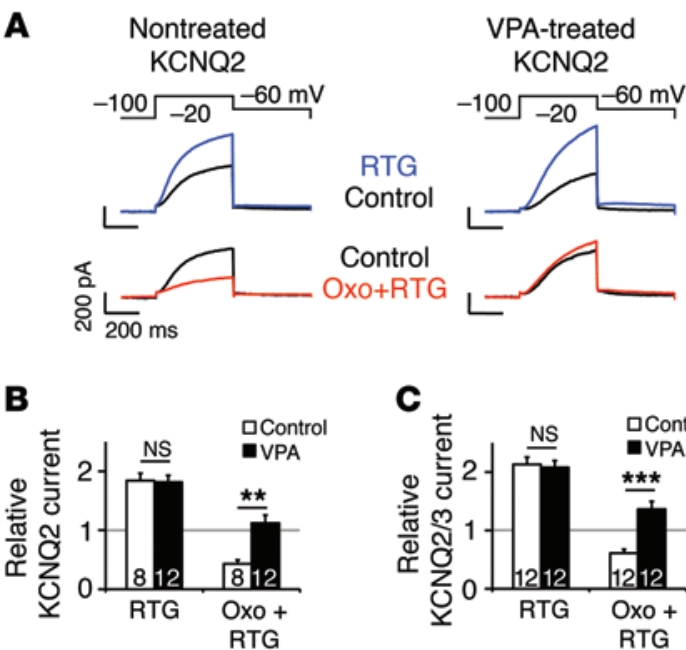

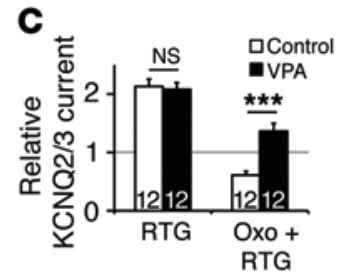

D
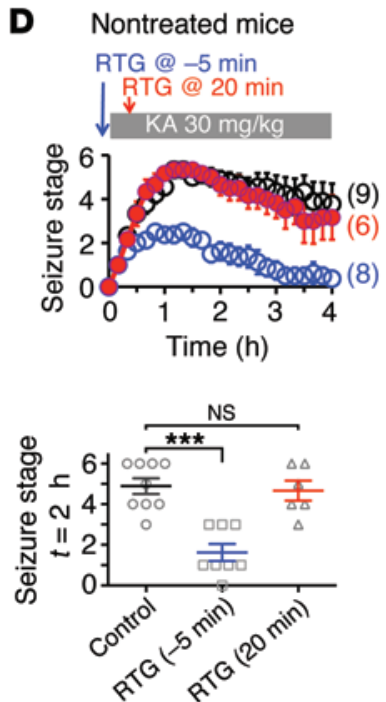

E VPA-treated mice

RTG@-5 min
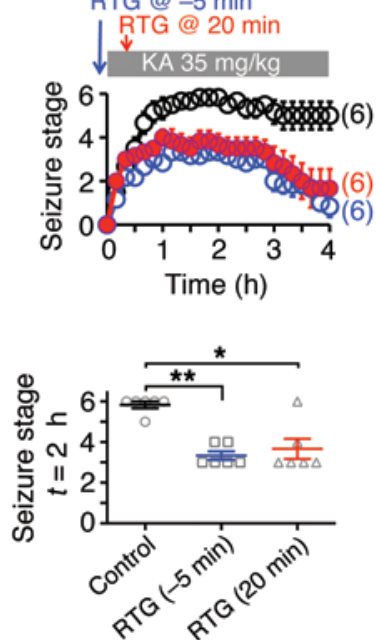

Figure 3. VPA protected retigabine-induced augmentation of KCNQ currents in the presence of oxo-M, as well as anticonvulsant effects with postseizure administration of retigabine. (A) Representative current traces showing $5 \mu \mathrm{M}$ retigabine-induced (RTG-induced) augmentation of KCNQ2 currents and its modulation by oxo-M. In VPA-nontreated $\mathrm{CHO}$ hm1 cells, $10 \mu \mathrm{M}$ oxo-M $+5 \mu \mathrm{M}$ retigabine-induced current suppression (left). In contrast, in VPAtreated cells, retigabine augmented KCNQ2 current even in the presence of $10 \mu \mathrm{M}$ oxo-M (right). (B) Summary of experiments shown in $\mathbf{A}$ for homomeric KCNQ2 channel. (C) Retigabine and oxo-M showed similar effects in heterologous KCNQ2/3 channels. Currents were normalized to the current before applying drugs. ${ }^{* *} P<0.01$ and ${ }^{* *} P<0.001$ using unpaired $t$ test. Numbers on bars represent $n$ values. (D) Retigabine (10 mg $\left./ \mathrm{kg}\right)$ showed anticonvulsant effects only when applied prior ( $t=-5 \mathrm{~min})$ to KA injection $(30 \mathrm{mg} / \mathrm{kg}, t=0)$. Lower panel shows seizure stages at $t=2$ hours. (E) Increased KA (35 mg/ $\mathrm{kg}$ ) was used to induce stage- 6 seizures in VPA-treated mice. In these VPA-treated mice, retigabine showed anticonvulsant effect even when applied after the induction of seizures. Lower panel shows seizure stages at $t=2$ hours. ${ }^{*} P<0.05,{ }^{* *} P<0.01$ and, ${ }^{* * *} P<0.001$ using Kruskal-Wallis one-way ANOVA followed by Dunn's multiple comparisons test were used. Error bars show \pm SEM.

siently expressed KCNQ2 channels in Chinese hamster ovary cells stably expressing human $\mathrm{m} 1$ muscarinic receptor, $\mathrm{CHO}$ hm1 cells (31). Homomeric KCNQ2 current was augmented by $5 \mu \mathrm{M}$ retigabine when applied alone, but when applied together with $10 \mu \mathrm{M}$ oxo-M, KCNQ2 current was decreased as reported (ref. 30 and Figure 3, A and B). In contrast, in $500 \mu \mathrm{M}$ VPA-treated $\mathrm{CHO}$ hm1 cells, KCNQ2 currents showed augmentation by retigabine even in the presence of $10 \mu \mathrm{M}$ oxo-M (Figure 3, A and B). Similar results were obtained from $\mathrm{KCNQ} 2 / 3$ heteromeric channels (Figure 3C), which are considered to be a common form of the endogenous M-channels. Retigabine application augmented KCNQ2/3 current. However, the current amplitude was reduced when applied together with oxo-M. Notably, KCNQ2/3 currents from VPA-treated cells showed augmentation by retigabine even in the presence of oxo-M. These results were consistent with our observation that VPA treatment preserves KCNQ current when $\mathrm{m} 1$ muscarinic receptor is activated. We then examined anticonvulsant effects of retigabine in kainate-induced seizures in mice. When retigabine was administered 5 minutes before application of kainate, it showed anticonvulsant effect on kainate-induced seizures, as expected (Figure 3D). However, it did not show any anticonvulsant effect when applied 20 minutes after kainate injection (Figure 3D), which suggests retigabine-sensitive M-channels are reduced after induction of seizures.

To test whether VPA modulates retigabine efficacy, we used an increased kainate dose $(35 \mathrm{mg} / \mathrm{kg})$, which induced stage- 6 seizures in VPA-treated mice (5/6 mice) (Figure 3E). Retigabine administration 5 minutes before kainate injection reduced seizure severity similarly as observed in VPA-nontreated mice. Impor- tantly, retigabine administration 20 minutes after kainate also reduced severity of seizures in VPA-treated mice (Figure 3E). These results further support that the anticonvulsant effect of VPA involves preservation of M-current during seizures.

Pharmacological modulation of behavioral seizure severity in the above experiments may merely reflect changes at the motor cortex without altering generalized seizure activity in other brain regions. Therefore, we evaluated accumulative neural hyperactivity in the brain during kainate-induced seizures by quantifying c-FOS expression in the hippocampus, a primary target brain region for administered kainate (32). We observed strong expression of c-FOS in the CA1 region and the dentate gyrus of the hippocampus 60 minutes after kainate injection, which would correspond to neuronal activity during status epilepticus in control mice, as reported previously (Figure 4 and refs. 33, 34). Kainate-induced c-FOS expression was reduced in VPA-treated mice. However, when XE991 was administered to VPA-treated mice, c-FOS expression showed equivalent induction, as compared with kainate alone (Figure 4). These results suggest that neuronal activity in the hippocampus correlates with behavioral seizures in our experimental conditions.

VPA interfered with the PKC-mediated pathway of muscarinic suppression of KCNQ2 current. Since VPA effects could be reconstituted in $\mathrm{CHO}$ hm1 cells expressing homomeric KCNQ2 channels (Figure 3, A and B), we used this heterologous expression system to further characterize the molecular process that is disrupted by VPA treatment. Overnight treatment with $500 \mu \mathrm{M}$ VPA reduced muscarinic suppression of $\mathrm{KCNQ} 2$ current (Figure $5 \mathrm{~A}$ ) similar to the effects observed in SCG neurons. Dose-response experiments 
A
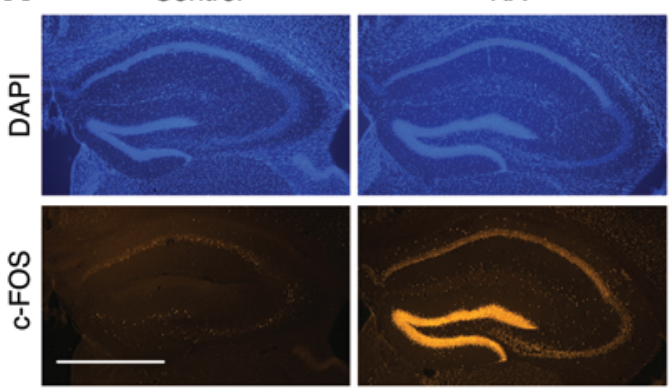

B

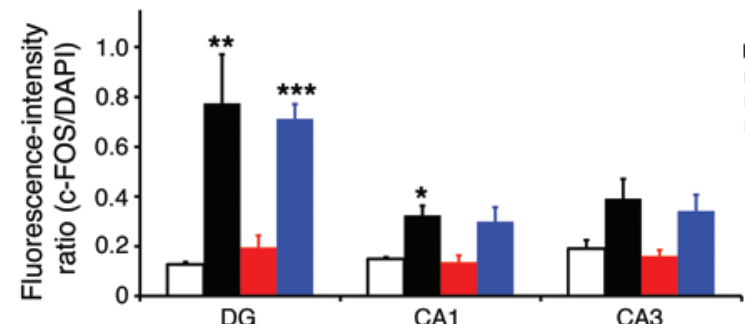

$V P A+K A$
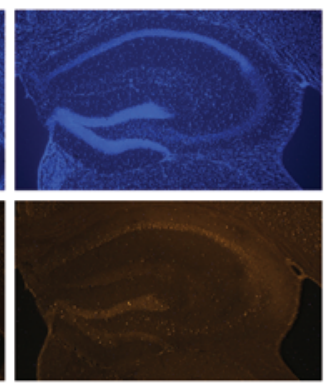

$V P A+K A+X E$

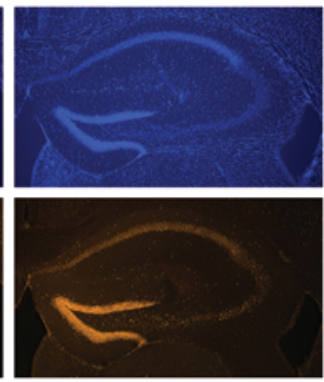

Control $(n=7)$

- KA $(n=8)$

- VPA + KA $(n=8)$

- VPA + KA + XE $(n=7)$

Figure 4. Induction of c-FOS expression in the hippocampus corresponded with behavioral seizures. (A) DAPI staining and c-FOS immunofluorescence staining in the hippocampus. Scale bar shows $1 \mathrm{~mm}$. (B) Histogram summarizing quantification of kainate-induced c-FOS expression. Reduced induction of c-FOS expression in the hippocampus was observed in VPA-treated mice. Restored induction of c-FOS was observed in VPA-treated mice coadministered with XE991 (2 mg/kg) and KA (30 mg/kg). Fluorescent intensities from corresponding regions of DAPI and c-FOS images were quantified and used to evaluate c-FOS expression relative to DAPI signals. ${ }^{*} P<0.05,{ }^{* *} P<0.01$, and ${ }^{* * *} P<0.001$ using Kruskal-Wallis one-way ANOVA followed by Dunn's multiple comparisons test. Error bars show \pm SEM.

showed that VPA disrupted muscarinic suppression of KCNQ2 current with $\mathrm{EC}_{50}$ of $213 \pm 9 \mu \mathrm{M}(n=7)$ (Figure $\left.5 \mathrm{~B}\right)$. Importantly, short exposure (5 minutes) to $500 \mu \mathrm{M}$ VPA did not alter muscarinic suppression of KCNQ2 current (Figure 5C). In addition, pretreatment with lithium chloride $(1 \mathrm{mM})$ or HDAC inhibitors $(1 \mathrm{mM}$ sodium butyrate or $2 \mu \mathrm{M}$ SAHA) did not affect muscarinic suppression of the KCNQ2 current (relative currents with oxo-M were $26.3 \pm 4.6 \%$ control [Figure $5 \mathrm{C}, n=8$ ], $23.5 \pm 5.6 \%$ lithium $[n=6$ ], $33.9 \pm 2.7 \%$ SAHA $[n=9], 27.0 \pm 5.8 \%$ butyrate $[n=9], P>0.05$ ).

We have previously demonstrated that PKC-mediated KCNQ2 subunit phosphorylation of serine 541 is important for suppression of the M-current (15). Therefore, we tested a mutant channel that lacks the phosphorylation acceptor residue, an alanine substitution of serine 541 in the KCNQ2 subunit: KCNQ2 $2^{\mathrm{S} 541 \mathrm{~A}}$. As we reported previously (15), KCNQ2 ${ }^{\mathrm{S} 541 \mathrm{~A}}$ exhibited smaller responses to oxo-M compared with the WT KCNQ2 current (Figure 5C). Importantly, VPA treatment did not further modify oxo-M responses of $\mathrm{KCNQ}^{\mathrm{S5}}{ }^{\mathrm{S} 1 \mathrm{~A}}$ current (Figure $5 \mathrm{C}$ ), which suggests that the KCNQ2 $2^{\mathrm{S5} 41 \mathrm{~A}}$ mutation occluded VPA effects.

Since PKC-mediated phosphorylation of KCNQ2 S541 triggers CaM dissociation from KCNQ2 channel (15), we measured KCNQ2-CaM dissociation by total internal reflection fluorescence Förster resonance energy transfer analysis (TIRF-FRET) using KCNQ2-mCitrine (KCNQ2-mCit) and CaM-mCerulean (CaM-mCer). Basal FRET efficiency showed positive FRET signals between KCNQ2-mCit and CaM-mCer, indicating association of these proteins, as we previously reported (15). These basal FRET efficiencies were not altered by VPA treatment $(0.18 \pm 0.02$ $[n=28]$ for VPA nontreated cells, $0.16 \pm 0.01[n=34]$ for VPA treated cells, $P=0.327, t$ test). Application of oxo-M reduced FRET signal in control cells, which corresponds to dissociation of CaM, as described (ref. 15 and Figure 5D). However, VPA treatment abolished oxo-M induced decrease in FRET efficiency (Figure 5D). These results support the hypothesis that VPA treatment disrupts $\mathrm{PKC}$-dependent KCNQ2 regulation.

Next, we asked whether this effect was due to the general inhibition of PKC activation or focal inhibition within the KCNQ2 channel complex. To address this question, we measured oxo-M induced cellular PKC activity using a cytosolic version of FRET based PKC kinase activity reporter, cytCKAR (35). Cellular PKC activity induced by muscarinic agonist was not altered by VPA treatment (Figure 5E), suggesting that interference of PKC signaling occurred only within the KCNQ2 channel complex.

Another key pathway of muscarinic suppression of the M-current is through PIP2 depletion. Therefore, we examined whether VPA treatment altered PIP2 depletion after muscarinic stimulation using CFP-PH, a CFP fusion protein with the $\mathrm{PH}$ domain of PLC $\delta$ (36). In control CHO hm1 cells, PIP2 depletion - triggered by $3 \mu \mathrm{M}$ oxo-M - induced translocation of CFP-PH into the cytoplasm (Figure 5F). VPA treatment did not evoke any changes in oxo-M-induced translocation of CFP-PH (Figure 5F). These results suggest that VPA selectively disrupted local PKC-mediated phosphorylation of the KCNQ2 channel without interfering with global PKC activation or phosphatidylinositol turnover.

VPA inhibited palmitoylation of AKAP15O and other neuronal proteins. AKAP79/150 anchors PKC to the KCNQ2 subunit and is critical for PKC-mediated KCNQ2 S541 phosphorylation $(14,18)$. Therefore, we next focused on AKAP79/150-dependent mechanisms. We first suspected that VPA decreased AKAP150 expression, since similar disruption of PKC-mediated KCNQ2 phos- 
A

$$
\text { Nontreated VPA-treated }
$$
KCNQ2(wt) KCNQ2(wt)
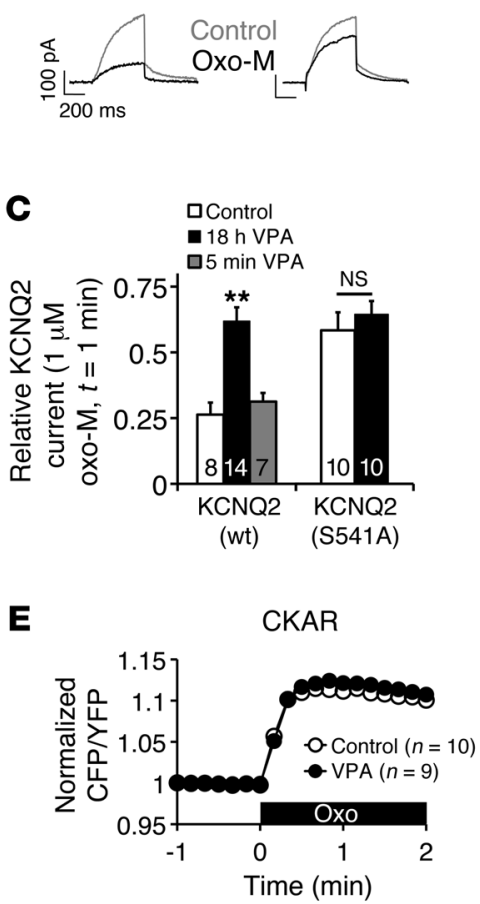

$\mathbf{F}$
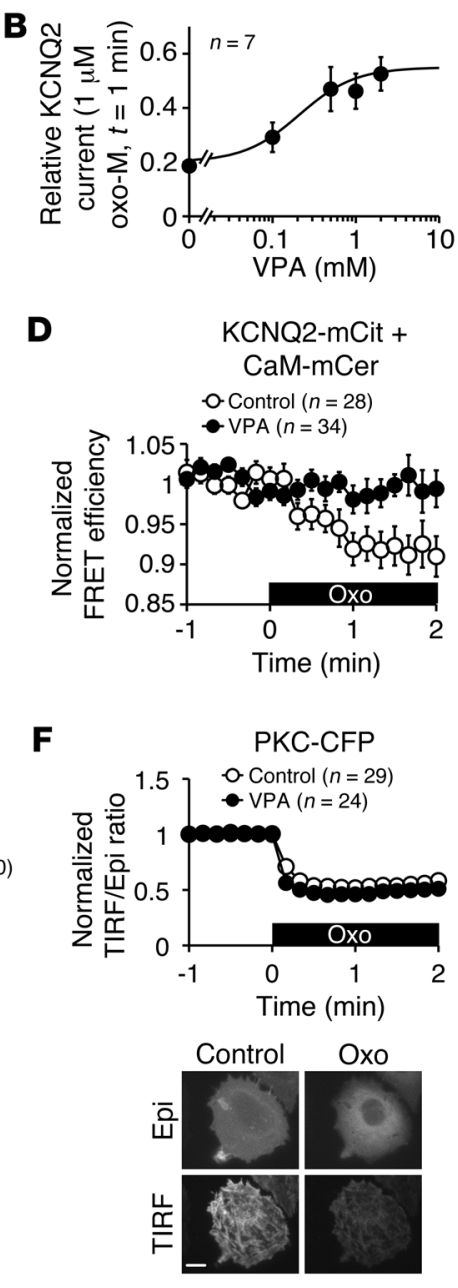

PKC-CFP

O. Control $(n=29)$
Figure 5. VPA disrupted PKC-mediated KCNQ2 regulation. (A) KCNQ2 current traces showing that $500 \mu \mathrm{M}$ VPA treatment attenuated $1 \mu \mathrm{M}$ oxo-M induced current suppression in KCNQ2 channels. (B) Dose-response curve for VPA against oxo-M induced KCNQ2 current suppression. (C) Relative KCNQ2 currents 1 min after oxo-M application. Eighteen-hour treatment with $500 \mu \mathrm{M}$ VPA reduced muscarinic suppression. Five-minute incubation with $500 \mu \mathrm{M}$ VPA did not attenuate oxo-M response. KCNQ2 ${ }^{5541 A}$ channels were more resistant to oxo-M. Notably, 18-hour treatment with $500 \mu \mathrm{M}$ VPA had no effect. Amplitudes of currents are relative to those before oxo-M application. ${ }^{*} P<0.01$ using Kruskal-Wallis one-way ANOVA followed by Dunn's multiple comparisons test. Numbers on bars represent $n$ values. (D) TIRF-FRET analysis between KCNQ2-mCit and CaM-mCer. Application of oxo-M decreased FRET signal corresponding to dissociation of CaM from KCNQ2 channel in control (open circle). VPA treatment prevented CaM dissociation (filled circle). Black box indicates presence of $3 \mu \mathrm{M}$ oxo-M. (E) Oxo-M induced PKC activity measured by FRET-based PKC probe, cytCKAR. VPA did not have any effect. Black box indicates presence of $3 \mu \mathrm{M}$ oxo-M. (F) PIP2 depletion measured by CFP-PH and $\mathrm{EPI} / \mathrm{TIRF}$ microscope. Oxo-M (3 $\mu \mathrm{M})$ induced translocation of CFP-PH. Fluorescent images are shown below for epi fluorescence (epi) and TIRF channel before (control) and after oxo-M (oxo) application. Scale bar: $10 \mu \mathrm{m}$. Error bars indicate \pm SEM. phorylation was observed in AKAP150-deficient cells $(18,19)$. However, VPA treatment did not change AKAP150 protein expression or protein interaction profiles of AKAP150 binding proteins (Supplemental Figure 2).

Accumulating evidence suggests that palmitoylation of AKAP150 is important for AKAP150-mediated signaling (20, 21). Therefore, we measured palmitoylation of AKAP150 by acylbiotin exchange (ABE) analyses (37) in cultured hippocampal neurons. This technique uses hydroxylamine $\left(\mathrm{NH}_{2} \mathrm{OH}\right)$ treatment to cleave palmitic acid from palmitoylated cysteine residues, followed by biotin labeling of newly exposed cysteine thiols. Therefore, biotin labeling of $\mathrm{NH}_{2} \mathrm{OH}$-treated samples indicates specific detection of palmitoylation. We observed that $1 \mathrm{mM}$ VPA treatment reduced palmitoylation of AKAP150 (Figure 6A, $P=0.038$, $n=3$, paired $t$ test). Interestingly, VPA treatment did not drastically alter the overall palmitoylation patterns in hippocampal neuron lysates (Figure 6B), suggesting some degree of selectivity for VPAsensitive palmitoylation.

We next examined the effects of in vivo administration of VPA on AKAP150 palmitoylation. After administering VPA (i.p., $250 \mathrm{mg} / \mathrm{kg} /$ day $\times 4$ days) to mice, cerebrums were collected and used for $\mathrm{ABE}$ analyses combined with immunoblots. Consistent with our results from cultured neurons, VPA treatment reduced palmitoylation of AKAP150 (Figure 6C). We also found that VPA suppressed palmitoylation of PSD95, flotillin-1, Fyn, and GRIP, but not EAAT2, confirming some degree of selectivity for VPAsensitive palmitoylation (Figure 6C).

Palmitoylation of AKAP150 is required for muscarinic regulation of the $M$-current. One remaining question was whether palmitoylation of AKAP79/150 is involved in muscarinic suppression of the M-current. We addressed this question by using AKAP150silenced SCG neurons. Endogenous AKAP150 was silenced in rat SCG neurons using a silencing plasmid, pSAKAP150i (18). In these AKAP150-silenced neurons, various versions of the human orthologue, AKAP79 - which are resistant to rat AKAP150 RNAi were expressed to examine whether they can restore AKAP79/150 signaling. AKAP150 silenced SCG neurons showed attenuated muscarinic responses, as we reported previously (ref. 18 and Figure 7). The muscarinic response was rescued by expression of WT AKAP79, as expected (Figure 7). Two cysteine residues in AKAP79, C36 and C129, have been identified as the palmitoylation sites $(20,21)$. Expression of the double serine-substituted mutant for the palmitoylation sites, AKAP79(C36S/C129S), could not restore the oxo-M response of KCNQ2 current (Figure 7).

It has been shown that myristoylation can mimic constitutive palmitoylation (38). Therefore, a myristoylation consensus site was added to the N-terminus of AKAP79(C36S/C129S), Myr-AKAP79(C36S/C129S). Expression of Myr-AKAP79(C36S/ 
A
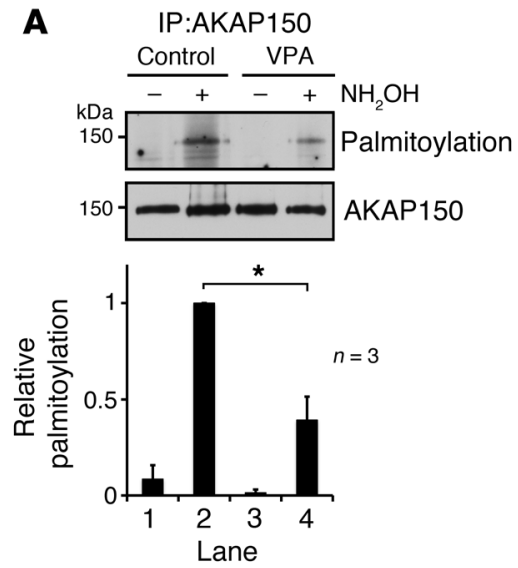

B

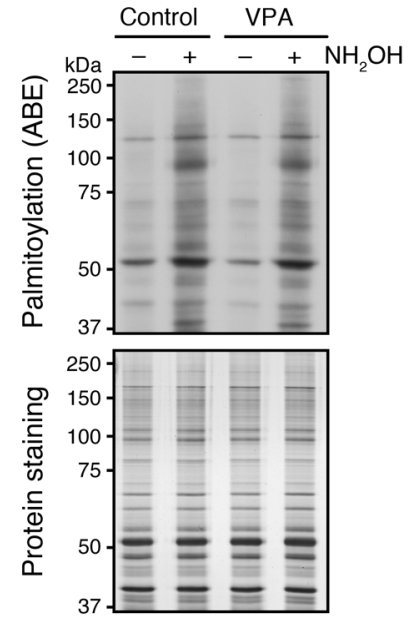

C
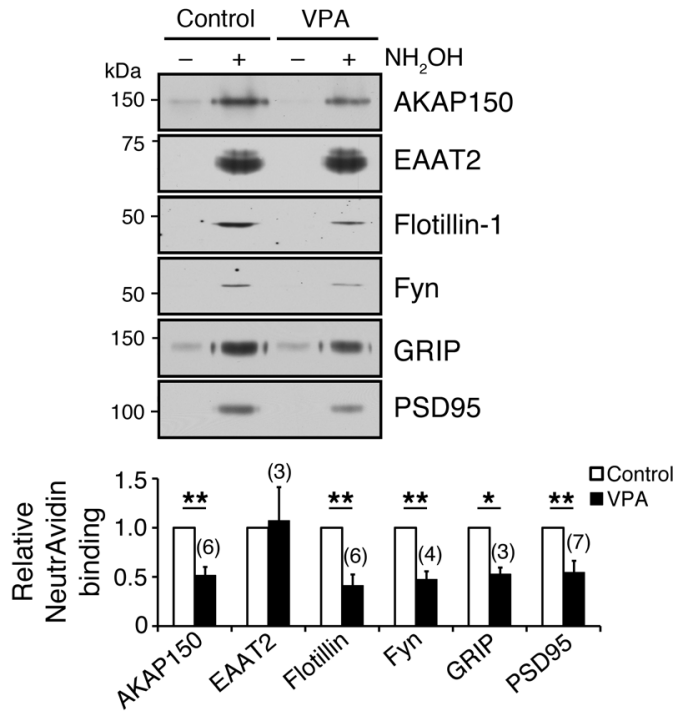

Figure 6. VPA selectively reduced palmitoylation of AKAP150 and several neuronal proteins. (A) Palmitoylation of AKAP150 was evaluated using cultured hippocampal neurons. After ABE labeling, AKAP150 protein was purified by IP using monoclonal anti-AKAP150 antibody. Palmitoylation was detected by NeutrAvidin-HRP (upper panel). Middle panel shows equal recovery of AKAP150 among treatments assessed by immunoblots using rabbit anti-AKAP150 antibody. Bottom histogram shows summary of 3 independent experiments. Palmitoylation of AKAP150 was reduced in VPA-treated neurons. Requirement of $\mathrm{NH}_{2} \mathrm{OH}$ for biotinylation verified selective labeling of palmitoylation. ${ }^{*} P<0.05$ using paired $t$ test to control. (B) VPA treatment had minimal effect on overall palmitoylation in cultured hippocampal neuronal lysates. (C) ABE labeling was performed on mouse brain extracts, followed by purification of labeled proteins by NeutrAvidin-beads. Palmitoylation of indicated neural proteins was assessed by immunoblotting. VPA reduced palmitoylation of several but not all neuronal proteins. ${ }^{*} P<0.05$ and ${ }^{*} P<0.01$ using paired $t$ test to control. Error bars show \pm SEM. Numbers above bars represent $n$.

C129S) restored muscarinic suppression of the M-current (Figure $7 \mathrm{~B})$. These results confirmed that palmitoylation of AKAP150 is critical for AKAP150-mediated regulation of the KCNQ2 channel.

AKAP150 mutants carrying a myristoylation site were resistant to VPA treatment. Since distinct enzymes regulate myristoylation and palmitoylation, we wondered if the myristoylated AKAP150restored M-channel pathway becomes resistant to VPA treatment. To test this, we overexpressed rat Myr-AKAP150(C36S/C123S), which corresponds to human Myr-AKAP79(C36S/C129S), in SCG neurons. Then, we treated neurons with $2 \mathrm{mM}$ VPA for 3 days and tested for muscarinic responses to $1 \mu \mathrm{M}$ oxo-M (Figure 8). In control experiments with overexpression of WT AKAP150, VPA pretreatment attenuated the muscarinic response of the M-current, as expected (Figure 8A). In contrast, muscarinic responses in MyrAKAP150(C36S/C123S)-expressing SCG neurons became insensitive to $2 \mathrm{mM}$ VPA (Figure 8, B and C). These results further support that VPA disrupts receptor-induced M-current suppression by interfering with palmitoylation of AKAP150.

\section{Discussion}

In the present study, we report that disruption of M-current suppression during seizure contributes to the nonacute anticonvulsant action of VPA. Conversely, we report that M-current suppression is involved in the pathophysiology of seizures. VPA has been demonstrated to have many pharmacological effects. However, some known effects require high concentrations of VPA. Therefore, it is important to evaluate effects at the concentration of VPA that is within the therapeutic range in humans $(28-700 \mu \mathrm{M})(1)$. We were able to demonstrate that VPA in this concentration range prevented muscarinic receptor-induced suppression of the M-current, as well as M-current-dependent hyperexcitability in primary neurons.

Previously, we showed that AKAP79/150 is important for receptor-induced suppression of the M-current $(14,18)$. In the present study, we demonstrated that pamitoylation of AKAP79/150 is critical for M-current regulation and that VPA disrupts AKAP79/150mediated M-current regulation by interfering with palmitoylation of AKAP79/150. Palmitoylation of AKAP79/150 has been shown to stabilize its membrane localization (20), which may be required during the rearrangement of the multiprotein KCNQ2-channel complex that occurs during muscarinic suppression of the M-current (15).

We also demonstrated that VPA suppressed palmitoylation of a minor fraction of neuronal proteins, including several synaptic proteins. Interestingly, kainate-induced seizures have been demonstrated to increase palmitoylation of PSD-95 and SNAP25, as well as AKAP150, which promotes synaptic potentiation (21). Therefore, in addition to VPA effects on the M-channel, inhibited palmitoylation of synaptic proteins may also contribute to the anticonvulsant action of VPA. Palmitoylation is mediated by palmitoyl acyltransferases, which consist of over 20 subtypes of DHHC (AspHis-His-Cys) proteins (23). GRIP1b has been shown to be palmitoylated by DHHC5 and DHHC8, while Fyn is palmitoylated by other DHHC enzymes (38). VPA suppressed palmitoylation of GRIP1 and Fyn to a similar degree, which indicates that VPA affects multiple DHHC subtypes. Therefore, VPA should be considered a palmitoyl acyltransferase inhibitor with moderate selectivity.

Although KCNQ subunit mutations have been linked to epilepsy, modulation of the WT M-channel has never been implicated in the pathophysiology of seizures. Our results suggest that M-cur- 


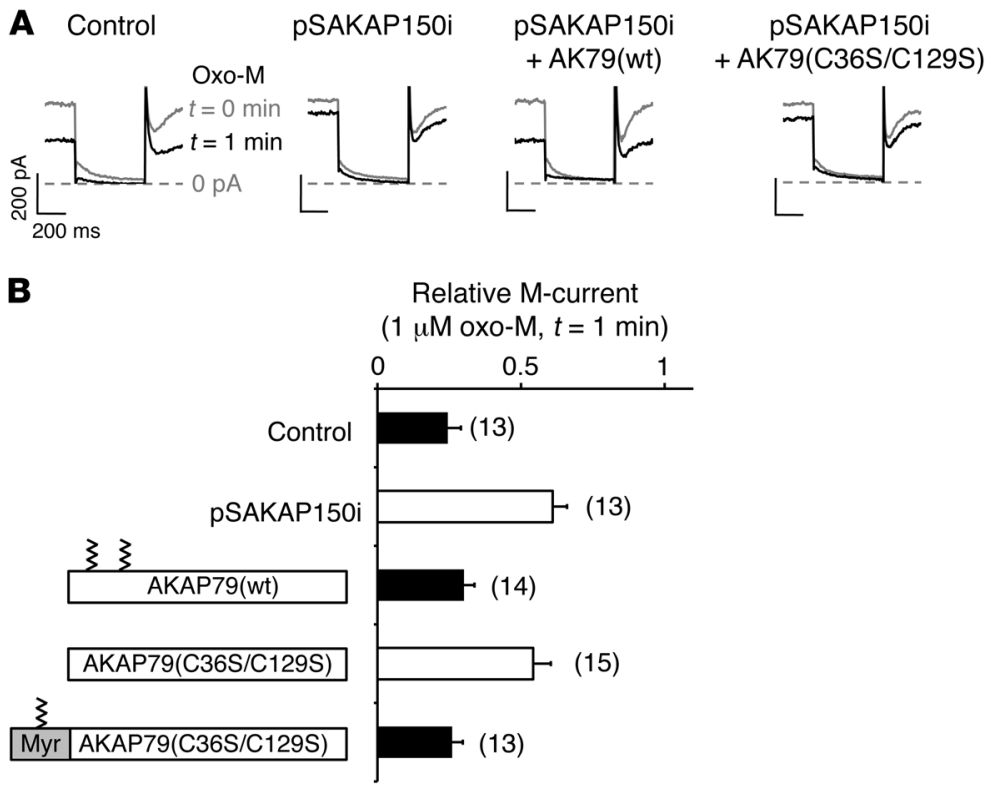

\begin{abstract}
Figure 7. Lipid modification of AKAP150 is required for muscarinic suppression of the M-current. (A) Representative current traces showing muscarinic response of the M-current in AKAP150-silenced rat SCG neurons. Silencing of endogenous AKAP150 attenuated $1 \mu \mathrm{M}$ oxo-M induced suppression of the M-current. Coexpression of WT AKAP79, AK79(wt), but not palmitoylation-deficient AKAP79(C365/C129S), AK79(C365/ C129S), rescued oxo-M induced suppression of the M-current. (B) Summary of the ability of AKAP79 mutants to restore muscarinic M-current suppression in AKAP150-silenced neurons. Open bars indicate $P<0.001$ from AKAP150-silenced control evaluated by one-way ANOVA followed by Dunnett's multiple comparisons test. AKAP79(C36S/C129S) did not restore AKAP150-mediated muscarinic signaling. Attachment of a myristoylation site to AKAP79(C36S/C129S) restored signaling. Error bars show \pm SEM. Numbers to the right of bars represent $n$.
\end{abstract}

rent is suppressed during seizures. XE991 is a voltage-dependent inhibitor that is effective only when the membrane potential is above $-65 \mathrm{mV}$ (28). Therefore, M-channels become sensitive to XE991 only when neurons are depolarized by neuronal activities, such as high-frequency firing. Indeed, we showed that XE991 aggravated kainate-induced seizures in VPA-treated mice. In contrast, XE991 administration did not show any effects in control kainate-induced seizures. This can happen if the M-current is already suppressed by seizure activity in affected regions, leaving few M-channels to be inhibited. Further support came from retigabine experiments. Retigabine has been shown to activate M-channels by shifting the activation voltage below the resting membrane potential range. However, retigabine-activated M-current can undergo receptor-induced suppression (30). Our experiments confirmed that retigabine could augment KCNQ2 or KCNQ2/3 currents when applied without a muscarinic agonist, but when applied together with a muscarinic agonist, muscarinic suppression overwhelmed retigabine-induced augmentation. However, when cells were pretreated with VPA, retigabine could augment $\mathrm{KCNQ} 2$ and $\mathrm{KCNQ} 2 / 3$ currents even in the presence of muscarinic agonist. In addition, efficacy of retigabine in kainate-induced seizures also supports this view. In nontreated mice, retigabine could suppress kainate-induced seizures only when applied before induction of seizures. In contrast, in VPA-treated mice, retigabine did not lose efficacy even when administered after seizure induction. These results suggest that administration of retigabine to VPA-treated patients would be a powerful procedure to cease status epilepticus. However, since M-current suppression has also been implicated in cognitive function, careful evaluation of long-term effects of this combination therapy would need to be performed. Together, these results suggest that M-current is suppressed during seizures and that VPA treatment preserves M-current by disrupting its receptor-induced suppression.

One scenario for M-current suppression during seizure is that seizures provoke excess neurotransmitter release that activates Gq-coupled receptors, thereby suppressing the M-current. Such seizure-induced M-current suppression increases the excitability of relevant neurons, which, in turn, facilitates propagation of ictal activities. Indeed, microdialysis studies have shown that several neurotransmitters - including acetylcholine $(39,40)$, serotonin $(39,41)$, noradrenaline $(41,42)$, and glutamate $(43,44)$ - are elevated during seizures. In addition, corresponding Gq-coupled receptors for these transmitters have been identified in hippocampal neurons: $\mathrm{m} 1$ acetylcholine receptor $(45,46)$, serotonin 5-HT2 receptor (47), $\alpha 1$ adrenergic receptor (47), and group 1 metabotropic glutamate receptor (48). However, noradrenaline and serotonin are considered to have anticonvulsant actions through distinct receptors $(49,50)$. Further studies are required to identify responsible neurotransmitters that suppress the M-current during seizures.

It has been reported that some VPA metabolites, which are primarily generated in the liver (51), have more potent anticonvulsant action (52). In our case, antihyperexcitation effects were reproduced in cultured cells. Therefore, we believe that VPA is the primary compound for the inhibition of palmitoylation in our conditions. However, we do not rule out the possibility that active VPA metabolites could be more potent palmitoylation inhibitors.

In summary, M-current suppression contributes to the pathophysiology of seizures, and preserved M-current during seizures is involved in the anticonvulsant effect of VPA, which is mediated by interfering with palmitoylation of AKAP79/150. These findings would provide insights and biochemical targets for developing better antiepileptic treatments.

\section{Methods}

Antibodies. Antibodies used for quantitative immunoblots were as follows: Anti-V5 epitope monoclonal antibody (catalog R960, Invitrogen); anti-flag M2 antibody, HA monoclonal antibody, and HA rabbit polyclonal antibody (catalogs F3165, H3663, H6908, Sigma-Aldrich); PSD-95 (catalog 041066, Millipore); flotillin-1, fyn, GRIP1, and EAAT2 (catalogs 041066, 610821, 611318, 611654, BD Biosciences); c-FOS (catalog PC38; Calbiochem); mouse monoclonal AKAP150 


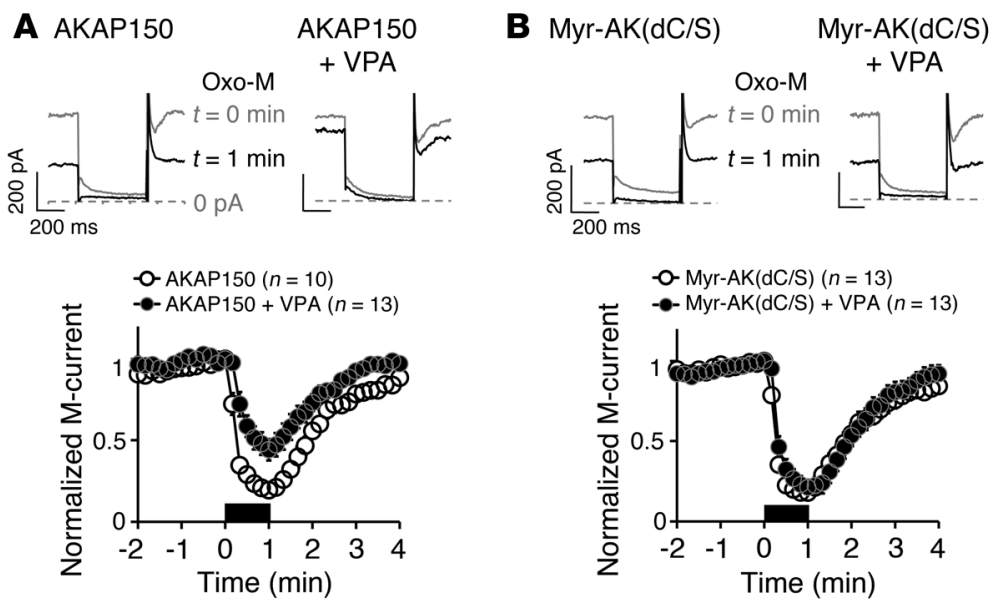

Figure 8. M-current suppression became resistant to VPA treatment when a myristoylated AKAP150 mutant was overexpressed in SCG neurons. (A) Current traces (top) and pooled results (bottom) showing oxo-M responses of the M-current in rat SCG neurons overexpressing WT AKAP150. VPA treatment ( $2 \mathrm{mM}$ ) attenuated responses to $1 \mu \mathrm{M}$ oxo-M. Black box indicates presence of oxo-M. (B) Current traces (top) and pooled results (bottom) showing oxo-M responses of the $\mathrm{M}$-current from SCG neurons overexpressing the myristoylation site-attached AKAP150(C36S/C123S), Myr-AK(dC/S). VPA treatment (2 mM) did not affect muscarinic response in Myr-AK(dC/S)-overexpressed neurons. (C) Histogram summarizing results shown in A and $\mathbf{B} .{ }^{* *} P<0.01$ using unpaired $t$ test with Welch's correction. Error bars show \pm SEM. Numbers above bars represent $n$.

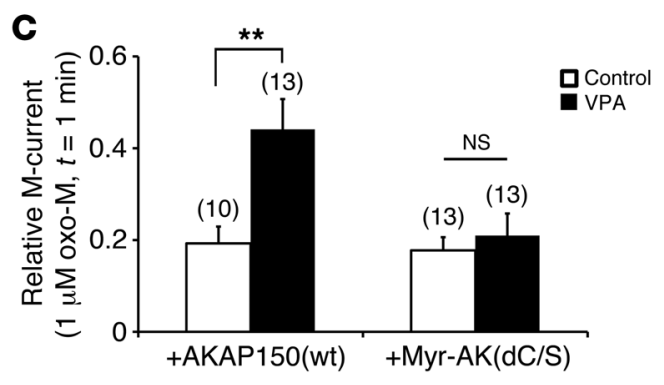

(catalog sc-377055; Santa Cruz Biotechnology Inc.); and Cy3-labeled goat anti-rabbit secondary antibody (catalog AP187C; Millipore). Rabbit polyclonal antibody for AKAP150 is a gift from John D. Scott (University of Washington, Seattle, Washington, USA) and has been described $(14,18,19,53)$.

Expression plasmids. The following expression plasmids used in this study have been described: $\mathrm{KCNQ} 2$ and its mutants $(14,15)$, AKAP79 and AKAP150 (18), PKCBII( $\Delta 1-32)-V 5$ (54), and cytCKAR (35, 54). The plasmid for CFP-PH was obtained from Tobias Meyer (Stanford University, Stanford, California, USA) via Addgene. HA-m1 muscarinic acetylcholine receptor was obtained from Missouri S\&T cDNA resource center. A myristoylation consensus sequence (MGQSLTT) (38) was added to the $\mathrm{N}$ terminus of AKAP150 by PCR to generate Myr-AKAP79(C36S/C129S) and Myr-AKAP150(C36S/C123S). All PCR-derived constructs were verified by sequencing.

Cell culture and VPA pretreatment. HEK293A (Invitrogen) cells were grown in DMEM with 10\% FBS. CHO hm1 (14) cells were grown in alpha-modified Eagle medium with 5\% FBS and $500 \mu \mathrm{g} / \mathrm{ml} \mathrm{G418}$ sulfate. SCG neurons were isolated from 14- to 19-day-old rats and cultured as described previously $(14,15,18)$. Rat neonatal hippocampal culture was prepared as described (18). VPA solution was prepared daily from powder and was added to the medium at $500 \mu \mathrm{M}$ unless indicated otherwise. A half volume of cultured medium was exchanged daily with freshly prepared VPA, vigabatrin, or phenytoin containing media and maintained for 3-4 days before recordings. For CHO hm1 cells, overnight incubation was sufficient to see VPA effects.

IP. HEK293A cells cultured in $10-\mathrm{cm}$ dishes were transiently transfected with LT1 transfection reagent (Mirus) with various expression plasmids. Cells were harvested 48 hours after transfection and lysed in $500 \mu \mathrm{l}$ HSE buffer $(150 \mathrm{mM} \mathrm{NaCl}, 5 \mathrm{mM}$ EDTA, $5 \mathrm{mM}$ EGTA, 20 mM HEPES [pH 7.4], 1\% Triton X-100, and complete pro- tease inhibitor cocktail [Roche Applied Science]). Supernatants were incubated with anti-flag antibody-conjugated resin or antibody and protein $\mathrm{G}$-conjugated resin. Following overnight incubation at $4^{\circ} \mathrm{C}$, immunoprecipitates were washed in HSE buffer. Bound proteins were analyzed by SDS-PAGE and immunoblotting.

Electrophysiological measurements and live cell imaging. Patchclamp recordings were performed at room temperature on isolated cells using an Axopatch $200 \mathrm{~B}$ patch-clamp amplifier (Molecular Devices). Signals were sampled at $2 \mathrm{kHz}$, filtered at $1 \mathrm{kHz}$, and acquired using pClamp software (version 7, Molecular Devices). Whole-cell patch-clamp technique was used for KCNQ2 current recording (15). Cells were perfused with a solution containing 144 $\mathrm{mM} \mathrm{NaCl}, 5 \mathrm{mM} \mathrm{KCl}, 2 \mathrm{mM} \mathrm{CaCl}, 0.5 \mathrm{mM} \mathrm{MgCl}_{2}, 10 \mathrm{mM}$ glucose, and $10 \mathrm{mM}$ HEPES (pH 7.4). Patch pipettes (2-4 M 2 ) were filled with intracellular solution containing $135 \mathrm{mM}$ potassium aspartate, $2 \mathrm{mM}$ $\mathrm{MgCl}_{2}, 3 \mathrm{mM}$ EGTA, $1 \mathrm{mM} \mathrm{CaCl}$, $4 \mathrm{mM}$ ATP, $0.1 \mathrm{mM} \mathrm{GTP}$, and 10 mM HEPES ( $\mathrm{pH}$ 7.2). KCNQ2 channels were activated from a holding potential of $-70 \mathrm{mV}$ by 2 -step test pulses to $0 \mathrm{mV}$ followed by $-60 \mathrm{mV}$ with $500 \mathrm{~ms}$ duration for each step. KCNQ2 currents were measured at the end of the $0 \mathrm{mV}$ step. For retigabine experiments, cells were held at $-100 \mathrm{mV}$; they were jumped to $-20 \mathrm{mV}$ and then to $-60 \mathrm{mV}$. For M-current in SCG as well as hippocampal neurons, perforated patch with amphotericin B was used as described $(14,18)$. Amplitudes of the M-currents were measured as deactivating currents during 500-ms test pulses to $-60 \mathrm{mV}$ from a holding potential of $-30 \mathrm{mV}$. For current clamp experiments, membrane potentials were adjusted to $-70 \mathrm{mV}$. During application of $20 \mu \mathrm{M}$ XE991, cells were held at $-30 \mathrm{mV}$ for 1 minute, then returned to $-70 \mathrm{mV}$. Live cell imaging was performed as described previously (15). Three filter methods were used to measure FRET between KCNQ2 and CaM as described (15). PKC-activity measurement using cytCKAR was conducted as described (54). 
Palmitoylation assay. ABE assay was performed following the protocol described by Wan et al. (37) with minor modifications. Briefly, cell and brain lysate were prepared in the presence of $10 \mathrm{mM}$ N-ethylmaleimide (NEM) followed by denaturation with chloroform/methanol precipitation. Treated samples were further incubated overnight with $10 \mathrm{mM}$ NEM. After extensive wash by chloroform/methanol precipitation, samples were subjected to $\mathrm{NH}_{2} \mathrm{OH}$ treatment. After each chloroform/methanol precipitation, protein pellets were solubilized in aqueous buffers by sonication. After wash, HPDP-biotin (Pierce Biotechnology) was used for sulfhydryl biotinylation. Biotin-exchanged samples were used for IP by mouse anti-AKAP150 antibody or affinity purification by NeutrAvidin resin (Pierce Biotechnology). Purified proteins were eluted by boiling in nonreducing SDS-PAGE loading buffer for detection with HRP-conjugated NeutrAvidin, or with dithiothreitol for detection with antibodies.

Kainate-induced seizure. For in vivo administration of VPA, adult C57BL/6 mice of both sexes (3-5 months old, The Jackson Laboratory) were administered (i.p.) either freshly prepared sodium VPA (250 $\mathrm{mg} / \mathrm{kg}$ ) or physiological saline solution twice daily for 4 days unless stated otherwise. For kainate-induced seizure experiments, $30 \mathrm{mg} / \mathrm{kg}$ kainate was injected (s.c.) 6 hours after the last VPA administration. One hour after kainate administration, mice were injected (i.p.) either with $2 \mathrm{mg} / \mathrm{kg}$ XE991 in Dulbecco's PBS (DPBS) or DPBS alone. Mice were video-taped for the subsequent 4 hours after kainate injection, and behavior was scored by treatment-blind observers every $10 \mathrm{~min}$ utes according to a modified Racine scale with the following criteria: (i) immobility; (ii) rigidity; (iii) automatisms with scratching, head bobbing, and circling; (iv) intermittent rearing and falling; (v) continuous rearing and falling; and (vi) tonic-clonic whole-body convulsions and rapid jumping. Animals that died during the experiments were assigned stage 6 thereafter.

c-FOS IHC. 1 hour after injection of kainate $(30 \mathrm{mg} / \mathrm{kg})$, mice were deeply anesthetized with isoflurane and perfused with $4 \%$ paraformaldehyde in PBS ( $\mathrm{pH}$ 7.4). Brains were then removed and postfixed with $4 \%$ paraformaldehyde in PBS ( $\mathrm{pH} 7.4$ ) overnight at $4^{\circ} \mathrm{C}$. Brains were sectioned into coronal slices $(30 \mu \mathrm{m})$ using a vibratome (VT1200, Leica Microsystems). Free-floating sections were washed with PBS in 24-well dishes and then incubated with PBS containing $0.3 \%$ hydrogen peroxide and $10 \%$ methanol for 10 minutes. After 3 washes with PBS, sections were incubated with a blocking buffer containing
$0.5 \%$ Triton X-100 and $1 \%$ normal goat serum in PBS. Sections were then incubated with primary antibody mixture containing rabbit antic-FOS antibody (1:1000 dilution) in the blocking solution for 48 hours at $4^{\circ} \mathrm{C}$. After 2 washes with PBS followed by 2 washes with Tris-buffered saline (TBS), sections were incubated with Cy3-conjugated anti-rabbit secondary antibody in TBS containing 0.5\% Triton X-100 and $1 \%$ normal goat serum for 2 hours at room temperature. After wash, sections were mounted onto glass slides using VECTASHIELD with DAPI (Vector Laboratories). Immunofluorescent images were acquired using a fluorescent light microscope (DM4000B, Leica Microsystems) equipped with a CCD camera (Optronics MicroFire, OPTMIF). Areas around the dentate gyrus, CA1 and CA3, were selected, and the average fluorescence intensity within each corresponding region of interest from DAPI and c-FOS staining images was quantified by observers blind to treatments using MetaMorph (Molecular Devices). Nucleus-free regions in the stratum oriens were used as a background for DAPI and c-FOS staining.

Statistics. Data are presented as mean \pm SEM. Statistical analyses were performed using GraphPad Prism. For comparing 2 groups, either unpaired or paired $t$ tests were used with Welch's correction. For experiments with more than 3 groups, one-way ANOVA or Kruskal-Wallis tests were used as follows. If variances were the same among groups - as evaluated by Bartlett's test and Brown-Forsythe test - one-way ANOVA, followed by Dunnett's multiple comparisons test, was used. If variances were different among samples, then Kruskal-Wallis test, followed by Dunn's multiple comparisons test, was used. When same cells received multiple treatments, then repeated-measures one-way ANOVA, followed by Tukey's multiple comparisons test, was used. $P$ values of less than 0.05 were considered significant.

Study approval. All animal experiments are approved by the Institutional Animal Care and Use Committee at the University of California, Irvine.

\section{Acknowledgments}

This work was supported by National Institutes of Neurological Disorders and Stroke (R01 NS067288) to N. Hoshi.

Address correspondence to: Naoto Hoshi, Department of Pharmacology, University of California, Irvine, 360 Med Surge II, Irvine, California 92697, USA. Phone: 949.824.0969; E-mail: nhoshi@uci.edu.
1. French JA, Pedley TA. Clinical practice. Initial management of epilepsy. $N$ Engl JMed. 2008;359(2):166-176.

2. McLean MJ, Macdonald RL. Sodium valproate, but not ethosuximide, produces use- and voltage-dependent limitation of high frequency repetitive firing of action potentials of mouse central neurons in cell culture. JPharmacol Exp Ther. 1986;237(3):1001-1011.

3. Nau H, Loscher W. Valproic acid: brain and plasma levels of the drug and its metabolites, anticonvulsant effects and gamma-aminobutyric acid (GABA) metabolism in the mouse. JPharmacol Exp Ther. 1982;220(3):654-659.

4. Phiel CJ, Zhang F, Huang EY, Guenther MG, Lazar MA, Klein PS. Histone deacetylase is a direct target of valproic acid, a potent anticonvulsant, mood stabilizer, and teratogen. J Biol Chem.
2001;276(39):36734-36741.

5. Jentsch TJ. Neuronal KCNQ potassium channels: physiology and role in disease. Nat Rev Neurosci. 2000;1(1):21-30.

6. Delmas P, Brown DA. Pathways modulating neural KCNQ/M (Kv7) potassium channels. Nat Rev Neurosci. 2005;6(11):850-862.

7. Soh H, Pant R, LoTurco JJ, Tzingounis AV. Conditional deletions of epilepsy-associated $\mathrm{KCNQ} 2$ and KCNQ3 channels from cerebral cortex cause differential effects on neuronal excitability. JNeurosci. 2014;34(15):5311-5321.

8. Miceli F, et al. Genotype-phenotype correlations in neonatal epilepsies caused by mutations in the voltage sensor of $\mathrm{K}(\mathrm{v}) 7.2$ potassium channel subunits. Proc Natl Acad Sci US A. 2013;110(11):4386-4391.

9. Rundfeldt C, Netzer R. The novel anticonvulsant retigabine activates $\mathrm{M}$-currents in Chinese hamster ovary-cells tranfected with human KCNQ2/3 subunits. Neurosci Lett. 2000;282(1-2):73-76.

10. Raol YH, Lapides DA, Keating JG, Brooks-Kayal AR, Cooper EC. A KCNQ channel opener for experimental neonatal seizures and status epilepticus. Ann Neurol. 2009;65(3):326-336.

11. Marrion NV. Control of M-current. Annu Rev Physiol.1997;59:483-504.

12. Gamper N, Shapiro MS. Calmodulin mediates $\mathrm{Ca}^{2+}$-dependent modulation of $\mathrm{M}$-type $\mathrm{K}^{+}$channels. J Gen Physiol. 2003;122(1):17-31.

13. Suh BC, Hille B. Recovery from muscarinic modulation of $\mathrm{M}$ current channels requires phosphatidylinositol 4,5-bisphosphate synthesis. Neuron. 2002;35(3):507-520.

14. Hoshi N, et al. AKAP150 signaling complex promotes suppression of the M-current by muscar- 
inic agonists. Nat Neurosci. 2003;6(6):564-571.

15. Kosenko A, et al. Coordinated signal integration at the M-type potassium channel upon muscarinic stimulation. EMBO J. 2012;31(14):3147-3156.

16. Suh BC, Hille B. PIP2 is a necessary cofactor for ion channel function: how and why? Annu Rev Biophys. 2008;37:175-195.

17. Wong W, Scott JD. AKAP signalling complexes: focal points in space and time. Nat Rev Mol Cell Biol. 2004;5(12):959-970.

18. Hoshi N, Langeberg LK, Scott JD. Distinct enzyme combinations in AKAP signalling complexes permit functional diversity. Nat Cell Biol. 2005;7(11):1066-1073.

19. Tunquist BJ, et al. Loss of AKAP150 perturbs distinct neuronal processes in mice. Proc Natl Acad Sci US A. 2008;105(34):12557-12562.

20. Delint-Ramirez I, Willoughby D, Hammond GV, Ayling LJ, Cooper DM. Palmitoylation targets AKAP79 protein to lipid rafts and promotes its regulation of calcium-sensitive adenylyl cyclase type 8. J Biol Chem. 2011;286(38):32962-32975.

21. Keith DJ, et al. Palmitoylation of A-kinase anchoring protein 79/150 regulates dendritic endosomal targeting and synaptic plasticity mechanisms. J Neurosci. 2012;32(21):7119-7136.

22. Resh MD. Fatty acylation of proteins: new insights into membrane targeting of myristoylated and palmitoylated proteins. Biochim Biophys Acta. 1999;1451(1):1-16.

23. Fukata Y, Fukata M. Protein palmitoylation in neuronal development and synaptic plasticity. Nat Rev Neurosci. 2010;11(3):161-175.

24. Kang R, et al. Neural palmitoyl-proteomics reveals dynamic synaptic palmitoylation. Nature. 2008;456(7224):904-909.

25. Belmaker RH. Bipolar disorder. N Engl J Med. 2004;351(5):476-486.

26. Borsotto M, et al. PP2A-Bgamma subunit and KCNQ2 K+ channels in bipolar disorder. Pharmacogenomics J. 2007;7(2):123-132.

27. Brown DA. Muscarinic acetylcholine receptors (mAChRs) in the nervous system: some functions and mechanisms. JMol Neurosci. 2010;41(3):340-346.

28. Romero M, Reboreda A, Sanchez E, Lamas JA. Newly developed blockers of the M-current do not reduce spike frequency adaptation in cultured mouse sympathetic neurons. Eur J Neurosci. 2004;19(10):2693-2702.

29. Wang HS, et al. KCNQ2 and KCNQ3 potassium channel subunits: molecular correlates of the M-channel. Science. 1998;282(5395):1890-1893.
30. Tatulian L, Delmas P, Abogadie FC, Brown DA. Activation of expressed KCNQ potassium currents and native neuronal M-type potassium currents by the anti-convulsant drug retigabine. J Neurosci. 2001;21(15):5535-5545.

31. Hoshi N, Langeberg LK, Gould CM, Newton AC, Scott JD. Interaction with AKAP79 modifies the cellular pharmacology of PKC. Mol Cell. 2010;37(4):541-550.

32. Ben-Ari Y, Cossart R. Kainate, a double agent that generates seizures: two decades of progress. Trends Neurosci. 2000;23(11):580-587.

33. Popovici T, Represa A, Crepel V, Barbin G, Beaudoin M, Ben-Ari Y. Effects of kainic acid-induced seizures and ischemia on c-fos-like proteins in rat brain. Brain Res. 1990;536(1-2):183-194.

34. Gall C, Murray K, Isackson PJ. Kainic acidinduced seizures stimulate increased expression of nerve growth factor mRNA in rat hippocampus. Brain Res Mol Brain Res. 1991;9(1-2):113-123.

35. Gallegos LL, Kunkel MT, Newton AC. Targeting protein kinase $\mathrm{C}$ activity reporter to discrete intracellular regions reveals spatiotemporal differences in agonist-dependent signaling. J Biol Chem. 2006;281(41):30947-30956.

36. Botelho RJ, et al. Localized biphasic changes in phosphatidylinositol-4,5-bisphosphate at sites of phagocytosis. J Cell Biol. 2000;151(7):1353-1368.

37. Wan J, Roth AF, Bailey AO, Davis NG. Palmitoylated proteins: purification and identification. Nat Protoc. 2007;2(7):1573-1584.

38. Thomas GM, Hayashi T, Chiu SL, Chen CM, Huganir RL. Palmitoylation by DHHC5/8 targets GRIP1 to dendritic endosomes to regulate AMPA-R trafficking. Neuron. 2012;73(3):482-496.

39. Zis AP, Nomikos GG, Brown EE, Damsma G, Fibiger HC. Neurochemical effects of electrically and chemically induced seizures: an in vivo microdialysis study in the rat hippocampus. Neuropsychopharmacology. 1992;7(3):189-195.

40. Baptista T, Weiss SR, Zocchi A, Sitcoske M, Post R. Electrical kindling is associated with increases in amygdala acetylcholine levels: an in vivo microdialysis study. Neurosci Lett. 1994;167(1-2):133-136.

41. Shouse MN, Staba RJ, Ko PY, Saquib SF, Farber PR. Monoamines and seizures: microdialysis findings in locus ceruleus and amygdala before and during amygdala kindling. Brain Res. 2001;892(1):176-192.

42. Kokaia M, Kalen P, Bengzon J, Lindvall O. Noradrenaline and 5-hydroxytryptamine release in the hippocampus during seizures induced by hippocampal kindling stimulation: an in vivo microdi- alysis study. Neuroscience. 1989;32(3):647-656.

43. Lehmann $\mathrm{A}$, Isacsson $\mathrm{H}, \mathrm{Hamberger} \mathrm{A}$. Effects of in vivo administration of kainic acid on the extracellular amino acid pool in the rabbit hippocampus. J Neurochem. 1983;40(5):1314-1320.

44. Pena F, Tapia R. Relationships among seizures, extracellular amino acid changes, and neurodegeneration induced by 4 -aminopyridine in rat hippocampus: a microdialysis and electroencephalographic study. J Neurochem. 1999;72(5):2006-2014.

45. Yamasaki M, Matsui M, Watanabe M. Preferential localization of muscarinic M1 receptor on dendritic shaft and spine of cortical pyramidal cells and its anatomical evidence for volume transmission. J Neurosci. 2010;30(12):4408-4418.

46. Martinello K, et al. Cholinergic afferent stimulation induces axonal function plasticity in adult hippocampal granule cells. Neuron. 2015;85(2):346-363.

47. Felder CC, Kanterman RY, Ma AL, Axelrod J. Serotonin stimulates phospholipase A2 and the release of arachidonic acid in hippocampal neurons by a type 2 serotonin receptor that is independent of inositolphospholipid hydrolysis. Proc Natl Acad Sci U S A. 1990;87(6):2187-2191.

48. Lujan R, Nusser Z, Roberts JD, Shigemoto R, Somogyi P. Perisynaptic location of metabotropic glutamate receptors mGluR1 and mGluR5 on dendrites and dendritic spines in the rat hippocampus. Eur J Neurosci. 1996;8(7):1488-1500.

49. Clinckers R, Smolders I, Meurs A, Ebinger G, Michotte Y. Anticonvulsant action of hippocampal dopamine and serotonin is independently mediated by D and 5-HT receptors. J Neurochem. 2004;89(4):834-843.

50. Giorgi FS, Pizzanelli C, Biagioni F, Murri L, Fornai $\mathrm{F}$. The role of norepinephrine in epilepsy: from the bench to the bedside. Neurosci Biobehav Rev. 2004;28(5):507-524.

51. Silva MF, et al. Valproic acid metabolism and its effects on mitochondrial fatty acid oxidation: a review. J Inherit Metab Dis. 2008;31(2):205-216.

52. Bialer M, Yagen B. Valproic Acid: second generation. Neurotherapeutics. 2007;4(1):130-137.

53. Dell'Acqua ML, Faux MC, Thorburn J, Thorburn A, Scott JD. Membrane-targeting sequences on AKAP79 bind phosphatidylinositol-4, 5-bisphosphate. ЕMBO J.1998;17(8):2246-2260.

54. Smith IM, Hoshi N. ATP competitive protein kinase $\mathrm{C}$ inhibitors demonstrate distinct state-dependent inhibition. PLoS One. 2011;6(10):e26338 